\title{
Tuning-Aided Implicit Space Mapping
}

\author{
Qingsha S. Cheng, ${ }^{1}$ John W. Bandler, ${ }^{1}$ José E. Rayas-Sánchez ${ }^{2}$ \\ ${ }^{1}$ Simulation Optimization Systems Research Laboratory, Department of Electrical and \\ Computer Engineering, McMaster University, Hamilton, Ontario, Canada L8S4K1 \\ 2 Department of Electronics, Systems and Informatics, Instituto Tecnológico y de Estudios Superiores \\ de Occidente (ITESO), Tlaquepaque, Jalisco 45090, Mexico
}

Received 25 June 2007; accepted 19 September 2007

\begin{abstract}
We demonstrate an implicit space mapping (ISM) method for microwave filter design that is enhanced and assisted by a tuning procedure. This procedure helps us to select design variables as well as suitable preassigned parameters for an ISM implementation. It also aids us in the convergence of our ISM algorithm. We investigated and solved a microstrip notch filter using this technique. This shows that tuning-aided sensitivity analysis guides the parameter selections and enhances the performance of ISM optimization. (C) 2008 Wiley Periodicals, Inc. Int J RF and Microwave CAE 18: 445-453, 2008.
\end{abstract}

Keywords: space mapping; EM simulator; circuit simulator; tuning; microstrip notch filter

\section{INTRODUCTION}

Space mapping (SM) technology [1] addresses the issue of reducing unnecessary time-consuming fullwave electromagnetic (EM) simulations of microwave structures in device modeling [2] and design optimization.

SM assumes the existence and exploitation of "fine" and "coarse" models. The "fine" model may be a CPU-intensive EM simulator that provides high accuracy. The "coarse" model is a simplified representation, typically an equivalent circuit with empirical formulas.

Implicit space mapping (ISM) has been demonstrated to work both in the design [3] and modeling [4] arenas. ISM [3, 4] optimization techniques exploit available preassigned parameters in the coarse model. The preassigned parameters are a set of parameters that are normally fixed during the design optimization

Correspondence to: Q. S. Cheng; e-mail: chengq@mcmaster.ca DOI 10.1002/mmce.20303

Published online 15 May 2008 in Wiley InterScience (www. interscience.wiley.com). process, such as dielectric constant and substrate height. Changing the values of some of these parameters, however, is expected to have a similar impact on the responses of the microwave components as does changing the designable parameters. In each iteration, we calibrate these preassigned parameters in the coarse model against the fine model (where the preassigned parameters are always fixed). A reoptimization is carried out on the calibrated coarse model (surrogate). The new design parameter values are then assigned to the fine model. These steps are repeated until the specifications are satisfied.

Tuning is a built-in functionality of Agilent ADS [5]. When a coarse model is designed in ADS, its parameters could be set as tunable. Each tunable parameter is provided with a slider bar. These slider bars can be moved up and down to alter the corresponding parameter values. The resulting graphic and numerical value changes in the responses are observed instantaneously.

In connection with ISM optimization, a question frequently asked is how to choose appropriate preassigned parameters for parameter extraction in the coarse model. We can use a tuning-aided ISM proce- 
dure to determine this. In the ADS coarse model, we tune the design and preassigned parameter candidates, e.g., substrate heights and dielectric constant values. This tuning allows us to note graphically how the parameters affect the responses. If we find out that one particular parameter tends to cancel the effect of varying certain design parameter(s), it could be a good ISM preassigned parameter. With tuning we can break down or split the uniformly-assigned parameters and examine their effects. For example, we can split the uniform substrate height into several individual heights, each height corresponding to that of one component of the microwave structure. These heights then can be tuned independently to determine if they are useful preassigned parameters for ISM. The splitting of preassigned parameters may generate a better match of the surrogate to the fine model during parameter extraction. In the same manner, we can use tuning to test if the design parameters can be split to create more flexibility in the coarse models and thus yield a better design solution. If the test result is true, we can split the fine model design parameters the same way as in the coarse model, and a better fine model solution is likely to be obtained.

Except for data from external fine models such as Sonnet $\boldsymbol{e m}$ [6], our tuning-aided SM process is implemented entirely in the Agilent ADS system. A microstrip notch filter is designed using this technique. In two iterations, an accurate design solution is obtained. The implementation takes full advantage of the Agilent ADS tuning technology.

\section{IMPLICIT SPACE MAPPING}

Our goal is to design a fine model

$$
\boldsymbol{x}_{f}^{*}=\arg \min _{x_{f}} U\left(\boldsymbol{R}_{f}\left(\boldsymbol{x}_{f}\right)\right)
$$

Here, the fine-model response vector is denoted by $\boldsymbol{R}_{f} \in \mathbb{C}^{m}$, e.g., $S_{11}$ at selected frequency points, where $m$ is the number of sample points. The fine-model design parameters are denoted $\boldsymbol{x}_{f} \in \mathbb{R}^{n}$, where $n$ is the number of design parameters. $U$ is a suitable objective function. For example, $U$ could be a minimax objective function with upper and lower specifications. Vector $\boldsymbol{x}_{f}^{*}$ denotes the optimal design to be determined.

We take the following model as our initial surrogate model

$$
\boldsymbol{R}_{s}\left(\boldsymbol{x}_{f}, \boldsymbol{x}_{0}\right)=\boldsymbol{R}_{c}\left(\boldsymbol{x}_{f}, \boldsymbol{x}_{0}\right)
$$

where $x_{0} \in \mathbb{R}^{p}$ represents the initial preassigned parameter vector and $p$ is the number of preassigned pa- rameters. Here, $\boldsymbol{R}_{s} \in \mathbb{C}^{m}$ and $\boldsymbol{R}_{c} \in \mathbb{C}^{m}$ represent the surrogate model and the coarse model response vectors, respectively. To match the surrogate to the fine model, in the $k$ th parameter extraction process, we extract the preassigned parameters

$$
\overline{\boldsymbol{x}}^{(\boldsymbol{k})}=\arg \min _{\boldsymbol{x}}\left\|\boldsymbol{R}_{f}\left(\boldsymbol{x}_{f}^{(\boldsymbol{k})}\right)-\boldsymbol{R}_{\boldsymbol{s}}\left(\boldsymbol{x}_{f}^{(\boldsymbol{k})}, \boldsymbol{x}\right)\right\|
$$

We then fix $\overline{\boldsymbol{x}}^{(k)}$ in the surrogate model and reoptimize the surrogate to obtain the next prediction of the fine model solution

$$
\boldsymbol{x}_{f}^{(k+1)}=\arg \min _{\boldsymbol{x}_{f}} U\left(\boldsymbol{R}_{s}\left(\boldsymbol{x}_{f}, \overline{\boldsymbol{x}}^{(k)}\right)\right)
$$

\section{TUNING-AIDED IMPLICIT SPACE MAPPING}

Tuning can help us in two ways when working with SM. It can be used for testing the sensitivity of the surrogate model responses to the design variables and/or preassigned parameters; and it can also be used to find a good starting point for direct surrogate model optimization and/or parameter extraction.

\section{A. Investigating the Surrogate Model Sensitivity to Parameters}

The sensitivity test using tuning determines how the responses vary with respect to changes in the design parameters. We expect to find that certain variables may significantly change the bandwidth and others shift the frequency. If two variables have the same effect on the response, it could mean too many degrees of freedom and we should probably keep one of the variables constant. If one variable is more sensitive than the other, we may want to retain the less sensitive one. Sometimes altering the value of a variable has a limited effect on the responses. This could result in insufficient flexibility in the model and thus we may be prevented from obtaining an optimal solution. We can consider using extra or split parameters. Tuning can indicate whether more flexibility is needed. Checking the objective function after optimization with extra or split parameters will tell us whether a model is actually more flexible. We expect that the inequality

$$
\begin{array}{r}
\min _{x_{f 1}, \ldots, x_{f(n+j)}}\left(U\left(\boldsymbol{R}_{s}\left(\left[x_{f 1}, x_{f 2}, \ldots, x_{f n}, x_{f(n+1)}, \ldots, x_{f(n+j)}\right]^{T}, \boldsymbol{x}\right)\right)\right. \\
\leq \min _{x_{f 1}, \ldots, x_{f n}}\left(U\left(\mathbf{R}_{s}\left(\left[x_{f 1}, x_{f 2}, \ldots, x_{f n}\right]^{T}, \boldsymbol{x}\right)\right)\right.
\end{array}
$$

holds, where the left-hand side of (5) has more flexibility than the right-hand side and $x_{f(n+1)}, \ldots, x_{f(n+j)}$ are $j$ split or extra parameters. 
To choose suitable preassigned parameters, the following criteria could be used.

$$
\begin{array}{r}
\| \boldsymbol{R}_{c}^{*}-\boldsymbol{R}_{c}\left(\overline{\boldsymbol{x}}_{f}+\left[0 \ldots, \Delta x_{f i}, \ldots 0\right]^{T}\right. \\
\left.\boldsymbol{x}_{0}+\left[0 \ldots,-\Delta x_{j}, \ldots 0\right]^{T}\right) \| \leq \varepsilon
\end{array}
$$

or

$$
\left\|\left(\boldsymbol{R}_{c}^{*}+\Delta \boldsymbol{R}_{c i}-\Delta \boldsymbol{R}_{c j}\right)-\boldsymbol{R}_{c}^{*}\right\| \leq \varepsilon
$$

where $\boldsymbol{x}_{0}$ is the default preassigned parameter value and $\overline{\boldsymbol{x}}_{f}$ and $\boldsymbol{R}_{c}^{*}$ are the optimal coarse model solution and the optimal coarse model responses, respectively. $\varepsilon$ is a user-specified small number. $\Delta \boldsymbol{R}_{c i}$ and $\Delta \boldsymbol{R}_{c j}$ are the response changes caused by the disturbance to the $i$ th element $\Delta x_{f i}$ in the design parameter vector and the $j$ th element $\Delta x_{j}$ in the preassigned parameter vector, respectively. The approximation tells us that for any small change $\left[0 \ldots, \Delta x_{f i}, \ldots 0\right]$ in the coarse model design parameters, we can find a corresponding change $\left[0 \ldots,-\Delta x_{j}, \ldots 0\right]$ in the preassigned parameter vector that tends to cancel out the change in responses. This means that changes in the design parameters can be compensated by changing certain preassigned parameters. For split design parameters, depending on their sensitivity, we may split a preassigned parameter to compensate each split design parameter individually or use a uniform preassigned parameter value to accommodate any changes in split design parameters. We can also use multiple or split preassigned parameters to restore changes caused by a single design parameter. In short, the preassigned parameters and design parameters do not have to be one-to-one pairs as long as the changes can be restored. In our implementation, ADS tuning is used to check the criterion (6).

\section{B. Finding a Better Starting Point for Optimization or Parameter Extraction}

In the surrogate model optimization and parameter extraction procedures, when the starting point is far from the solution, the optimization engine may be trapped in a local minimum. We can use parameter tuning to move the starting point closer to a better solution. Especially in the case of frequency misalignment, a simple shift of the response using tuning can help the parameter extraction process escape from a local minimum. Normally, a shift in the dielectric constant can move the response to a good starting point. We call this procedure a "jump-start." The process is also workable for coarse model optimization or surrogate model optimization.

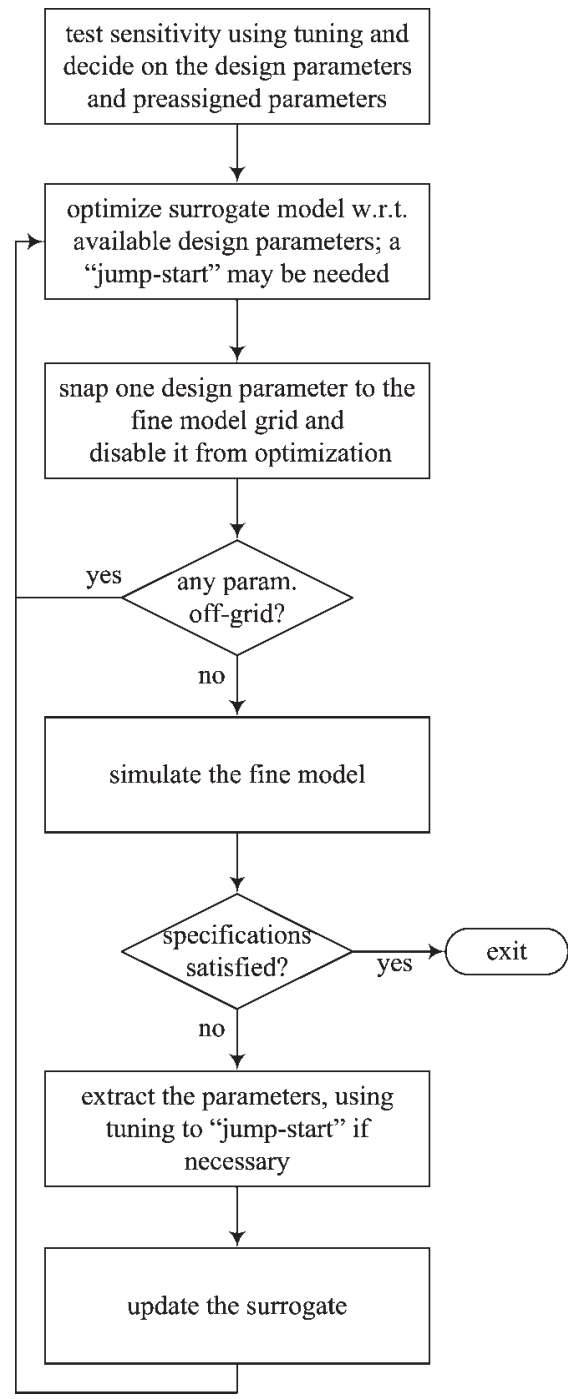

Figure 1. Flowchart for tuning-aided ISM optimization.

In Figure 1, we show the flowchart of our tuningaided ISM procedure. We start by investigating the sensitivity using available tuning. The design parameter values and preassigned parameter values are determined according to (5) and (6). We optimize the surrogate model with respect to the design parameters, jump-starting the process using tuning, if necessary. If the fine model is snapped to a grid, we need to supply the fine model with on-grid parameters. We then snap one design parameter to the grid in the surrogate model. The snapping procedure disturbs the responses. We then optimize the surrogate model again with the snapped parameter fixed. Then we snap the second design parameter to the grid and reoptimize. We proceed in this fashion until all the design parameters are snapped to the grid. These 


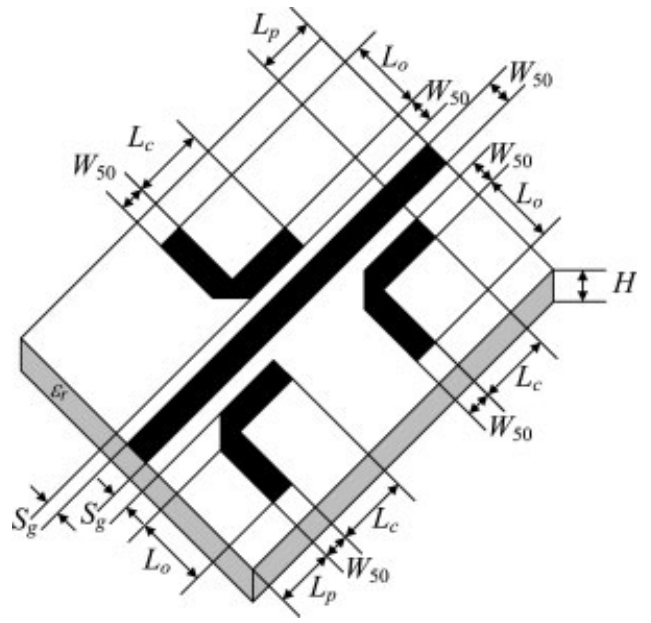

Figure 2. Microstrip notch filter with mitered bends [7].

design parameter values are then supplied to the fine model. After an accurate on-grid fine model simulation is obtained, we proceed with parameter extraction. We can now tune and extract the preassigned parameters so that the surrogate is aligned with the fine model. The tuning is especially necessary at the first iteration of SM optimization, when the fine model is far from the initial surrogate model. The extracted preassigned parameter values are fixed in the surrogate. The new surrogate is then reoptimized with respect to the original design parameters.

\section{MICROSTRIP NOTCH FILTER EXAMPLE}

We use a microstrip notch filter [7] example to illustrate the tuning-aided ISM design optimization procedure.
The parameterized dimensions of the filter are shown in Figure 2. $L_{o}$ is the length of the open stubs, $L_{c}$ is the length of the coupled lines, and $S_{g}$ is the separation gap. The width $W_{50}$ is the same for all the sections as well as for the input and output lines, of length $L_{p}$. A substrate is used with thickness $H$ and relative dielectric constant $\varepsilon_{r}$. The initial optimization variables are $\boldsymbol{x}_{f}=\left[L_{c} L_{o} S_{g}\right]^{T}$. The remaining parameter values are $H=10 \mathrm{mil}, W_{50}=31 \mathrm{mil}, \varepsilon_{r}=2.2$ (RT Duroid 5880, with loss tangent $=0.0009$ ). The design specifications are $\left|S_{21}\right| \leq 0.05$ in the stopband and $\left|S_{21}\right| \geq 0.95$ in the passbands, where the stopband lies between $13.19 \mathrm{GHz}$ and $13.21 \mathrm{GHz}$, and the passband includes frequencies below $13 \mathrm{GHz}$ and above $13.4 \mathrm{GHz}$.

\section{A. Coarse Model}

The coarse model is implemented in Agilent ADS as shown in Figure 3. It uses the built-in ADS microstrip circuit models available for transmission lines, coupled lines, and bends. The coarse model uses $L_{p}$ $=31 \mathrm{mil}$.

\section{B. Fine Model}

The fine model implementation is in Sonnet $\boldsymbol{e m}$ (see Fig. 4). We use a cell size of $0.5 \mathrm{mil} \times 0.5 \mathrm{mil}$, with $H_{\text {air }}=60$ mil (see the $3 \mathrm{D}$ view in Fig. 5). All the metals, including Sonnet's metallic box, are considered lossless. We optimize the coarse model using the Agilent ADS minimax method.

In the ADS coarse model, we make variables $L_{c}$, $L_{o}, S_{g}$, substrate height $H$, and dielectric constant $\varepsilon_{r}$ tunable. We can now move the tune slider bars up and down to see how the response changes with the variables. The first observation is that $L_{c}$ and $L_{o}$ have

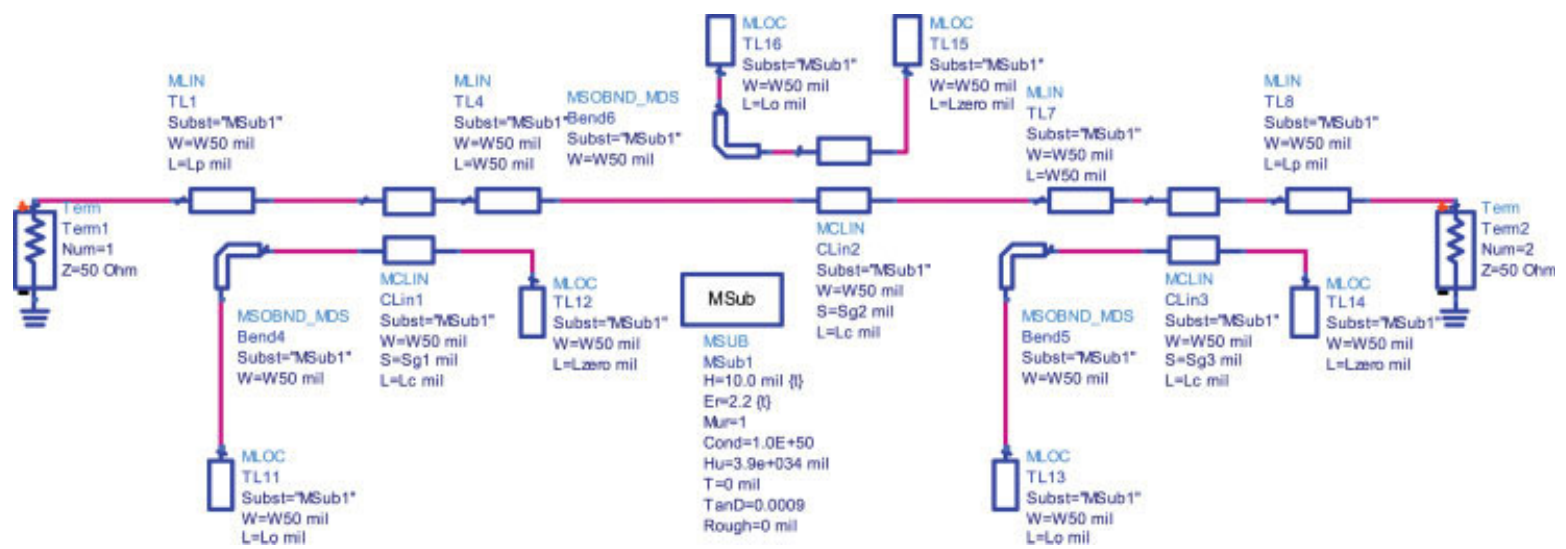

Figure 3. Agilent ADS coarse model of the microstrip notch filter. [Color figure can be viewed in the online issue, which is available at www.interscience.wiley.com.] 


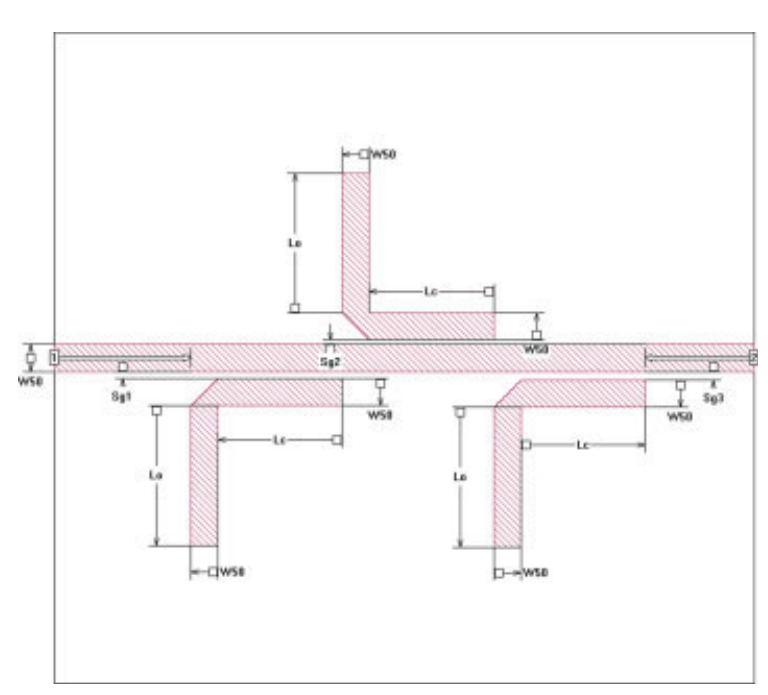

Figure 4. Sonnet $\boldsymbol{e m}$ fine model. [Color figure can be viewed in the online issue, which is available at www. interscience.wiley.com.]

an almost identical effect on the responses; they both shift the frequency. So we fix $L_{o}$ at $160 \mathrm{mil}$ and use $L_{c}$ as a design variable. Now we tune the design variable $S_{g}$. It verifies that $S_{g}$ controls the bandwidth of the responses. Our tuning experiment shows that its effect on the bandwidth is limited and this may result in low attainability of a good solution. Since $S_{g}$ affects the coupling between the resonators, we decide to split $S_{g}$ into the three variables $S_{g 1}, S_{g 2}$, and $S_{g 3}$ and assign them to the microstrip gaps in each subsection, as shown in Figure 3, $S_{g 1}$ for the first microstrip coupled line "CLin1," $S_{g 2}$ for "CLin2," and $S_{g 3}$ for "CLin3." With three variables instead of one, the bandwidth can vary over a larger range. We conducted an experiment to show the bandwidth change. In our experiment, the coarse model is opti-

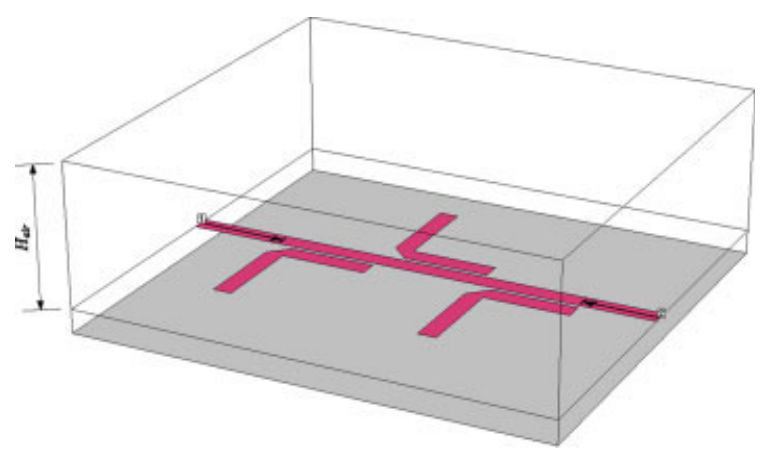

Figure 5. Sonnet $\boldsymbol{e m}$ fine model in $3 \mathrm{D}$ view. [Color figure can be viewed in the online issue, which is available at www.interscience.wiley.com.] mized using the split $S_{g}$ and uniform $S_{g}$, against the original specification. A better performance is obtained for the split $S_{g}$ (Table I). We double the bandwidth of the stopband specification while keeping the passband specification intact. Again we obtain a better solution (shown in Table I). The experiment tells us that splitting $S_{g}$ yields a more flexible model and a better chance of convergence to a good solution. This conclusion should be true for the fine model too.

The original design methodology [7, 8], i.e., the design using uniform $S_{g}$, trades the flexibility of the design for fewer design variables. More design variables normally mean a more difficult direct optimization or tuning of the fine model. This is the justification for using a minimal number of design variables. In SM technology, however, the optimization burden is shifted to the coarse model, so that increasing flexibility (using more design variables) will introduce less difficulty to the problem than direct optimization or tuning of the fine model. It is justifiable to use more variables for SM in which the added flexibility increases the probability of obtaining a good coarse model solution and thereby a better final fine model solution.

We can see the effect of the preassigned parameters using the ADS tuning capability too. By moving the slider bars of the Tune Parameters dialogue box, see Figure 6, we determine that the dielectric constant $\varepsilon_{r}$ can compensate for the frequency shift and that the substrate height $H$ can compensate for the bandwidth change. While this may be an advantage sometimes, here there seems no need for extra or split preassigned parameters.

The SM scheme is then determined as follows. The design parameters are $L_{c}, S_{g 1}, S_{g 2}$, and $S_{g 3}$. We use ISM with preassigned parameters $\varepsilon_{r}$ and $H$ (uniform in the whole structure).

We show the initial (on-grid) coarse model and fine model solutions in Figure 7. In two iterations (three fine model simulations), a good solution is obtained. Figure 8 shows a comparison between the results obtained by our method and those obtained by

TABLE I. Specification Errors for Coarse Model Optimization

\begin{tabular}{lcc}
\hline & $\begin{array}{c}\text { Original } \\
\text { Specification }\end{array}$ & $\begin{array}{c}\text { Doubled } \\
\text { Stopband } \\
\text { Bandwidth }\end{array}$ \\
\hline Uniform $S_{g}$ Splitting $S_{g}$ into & -0.03063 & -0.01639 \\
$\quad S_{g 1}, S_{g 2}$, and $S_{g 3}$ & -0.03695 & -0.02951 \\
\hline
\end{tabular}




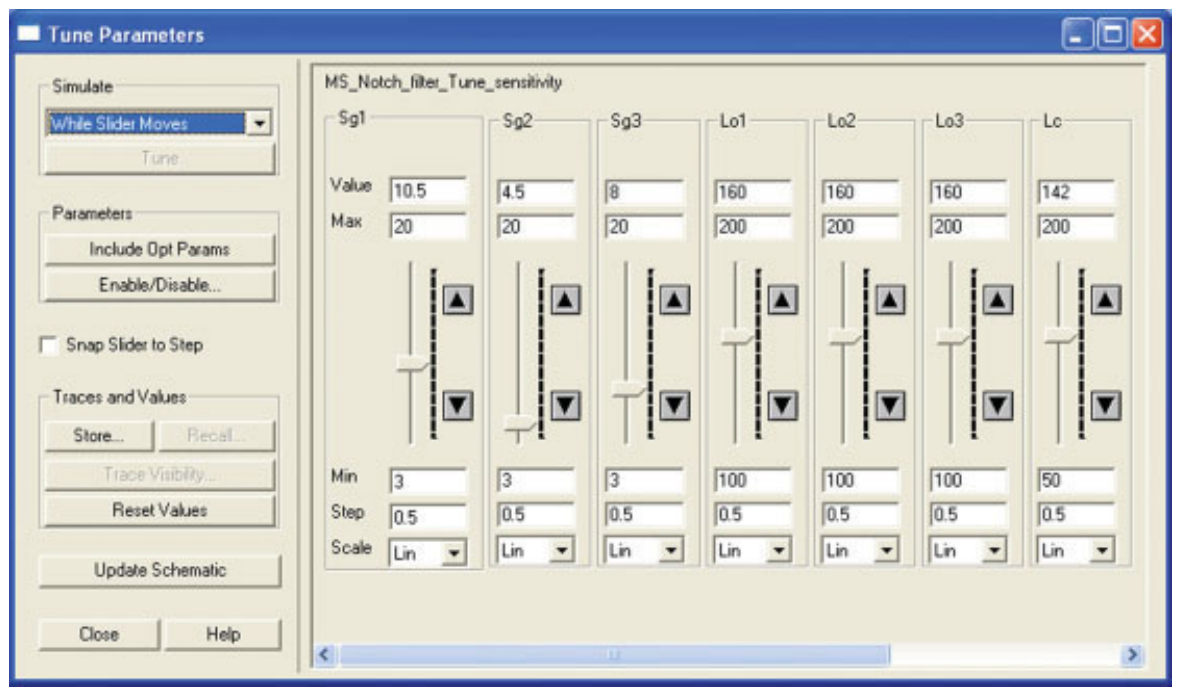

Figure 6. Agilent ADS tuning parameter dialogue box. [Color figure can be viewed in the online issue, which is available at www.interscience.wiley.com.]

applying a constrained Broyden-based linear-input $\mathrm{SM}$ algorithm [7] to the same problem (the coarse model used in [7] is implemented in APLAC; APLAC Version 7.91, APLAC Solutions Corporation, Finland, 2004). A detailed stopband fine model simulation is shown in Figure 9. It is seen that our approach yields a wider stopband in both the surrogate (coarse) model and the fine model, because of the introduction of nonuniform separation gaps $\left(S_{g 1}\right.$, $S_{g 2}$, and $S_{g 3}$ ). We show the evolution of the specification error in Figure 10. Table II summarizes the solutions obtained by both methods.

As soon as we obtained a good final solution using our tuning-aided ISM optimization, the final surrogate is now available as a better surrogate than the original coarse model. It can be used for other pur-

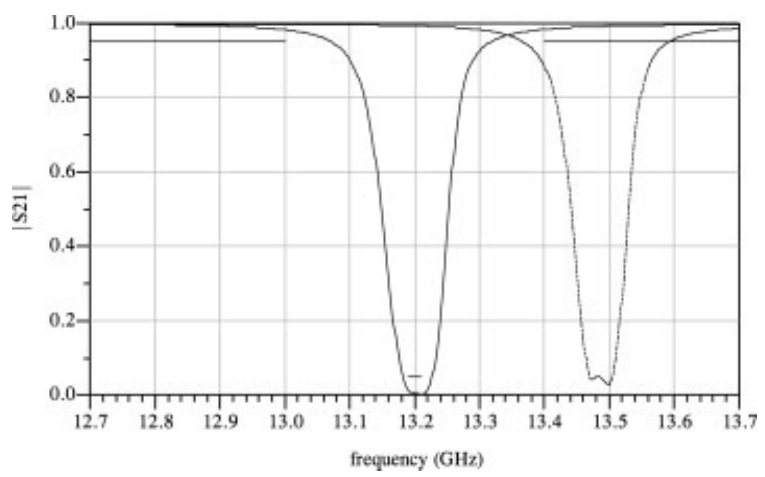

Figure 7. Coarse (-) and fine (---) model responses at the initial optimal coarse model solution. Note that the coarse model used in [7] is closer to the fine model than the coarse model used in this work. poses such as yield estimation and optimization. We run an ADS yield estimation on the final surrogate using a uniform distribution of \pm 0.2 mil for each parameter. After estimating 1250 random points, a yield of $97.4 \%$ is obtained. We now apply yield optimization using ADS. With only minor changes in the parameters, the ADS yield optimization engine is able to drive the yield to $100 \%$ (Fig. 11). The optimal yield solution is $\left[L_{c}, S_{g 1}, S_{g 2}, S_{g 3}\right]^{T}=[142.09,10.43$, $4.52,8.01]^{T}$.

\section{DISCUSSION ON TUNING}

Tuning is a commonly used engineering procedure. In our approach, we use tuning to aid SM to find

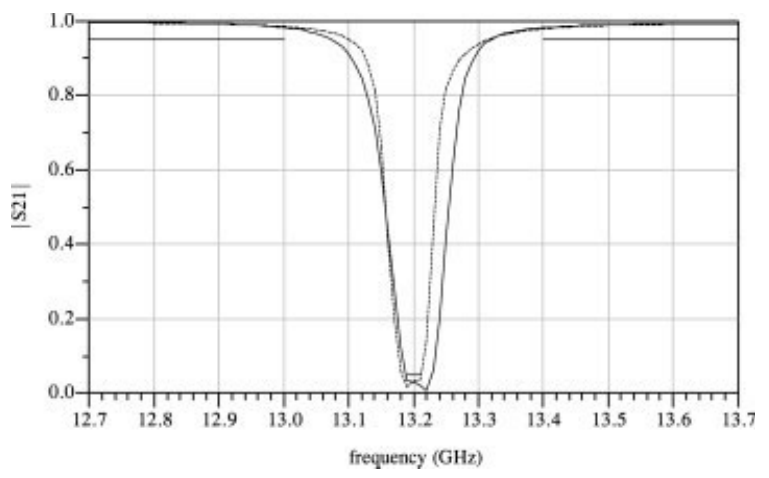

Figure 8. Fine model optimal response comparison between the tuning-aided ISM optimization (-) and the constrained Broyden-based linear-input SM approach (---) [7]. 


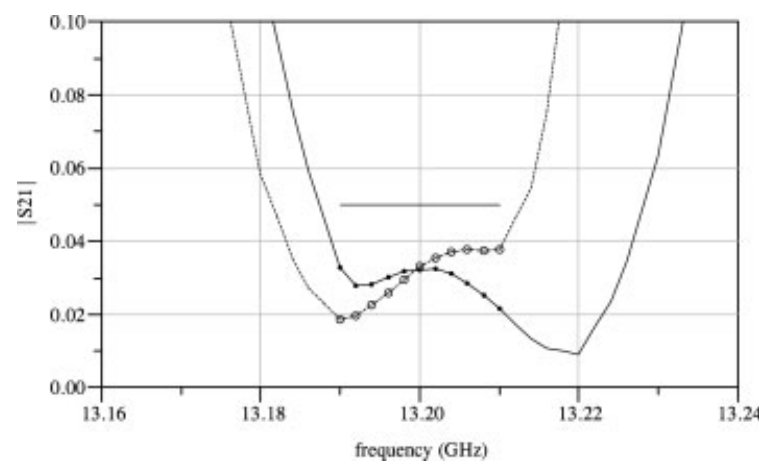

Figure 9. Fine model optimal response stopband detail comparison between the tuning-aided ISM optimization (-) and the constrained Broyden-based linear-input SM approach (---) [7].

appropriate design variables and implicit preassigned parameters. It also helps in the convergence of the ISM optimization algorithm. Since other space-mapping related methods can be seen as special cases of ISM [9], tuning can be of assistance in all types of SM too.

Tuning is widely applied in the microwave and RF component and device design process. For example, Rautio [10] discussed a tuning methodology to design an LTCC quad band receiver, and Swanson and Wenzel [11] presented a comb-line filter design using a circuit simulator tuning. Their idea is to obtain multiport $S$-parameters from EM simulation results of a structure with tuning ports. The obtained $S$-parameters form an $S$-parameter file component with ports. The tuning ports of the $S$-parameter file component are connected with appropriate variable circuits. This circuit-appended $S$-parameter file model is then optimized in a circuit simulator with respect to the added circuit parameter values to satisfy the design specifications. The parameter values are converted back to corresponding dimension values of the structure in the EM simulator.

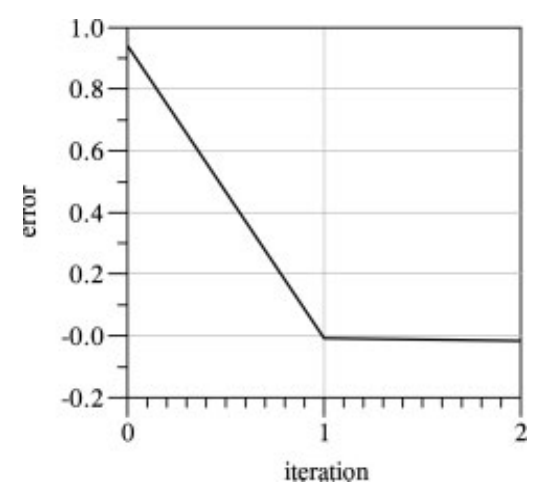

Figure 10. Evolution of the tuning-aided ISM specification error.
TABLE II. Solution Comparison Between the TuningAided Implicit Space Mapping and the Constrained Broyden-Based Linear-Input Space Mapping

\begin{tabular}{lccccc}
\hline & $\begin{array}{c}\text { After } \\
\text { Snitial } \\
\text { Solution }\end{array}$ & $\begin{array}{c}\text { First } \\
\text { Iteration }\end{array}$ & $\begin{array}{c}\text { After } \\
\text { Second } \\
\text { Iteration }\end{array}$ & $\begin{array}{c}\text { Initial } \\
\text { Solution } \\
{[7]}\end{array}$ & $\begin{array}{c}\text { Final } \\
\text { Solution } \\
\text { [7] }\end{array}$ \\
\hline Error & 0.942 & -0.009 & -0.017 & - & -0.012 \\
$L_{c}$ & 135 & 142 & 142 & 143 & 143.5 \\
$L_{o}$ & 160 & 160 & 160 & 158 & 159 \\
$S_{g 1}$ & 9 & 8 & 8 & 8 & 8 \\
$S_{g 2}$ & 5 & 5 & 4.5 & 8 & 8 \\
$S_{g 3}$ & 10 & 10 & 10.5 & 8 & 8 \\
\hline
\end{tabular}

If we assume the circuit-appended $S$-parameter file model is an enhanced coarse model (or surrogate) and the EM simulation of the structure is the fine model, we can see the similarity between their tuning methods and our tuning-aided SM. In both methods, tuning is applied in a circuit simulator and the optimization of the circuit model is used as a way of determining values for the design parameters. The design parameter values are then applied to the fine model.

A sensitivity analysis option in the ADS optimization algorithm is available. It may be used for our further investigation of the possibility of automating the preassigned parameter selection process, i.e., finding a set of preassigned parameters that has a cancel-out effect on certain design parameters automatically using sensitivity information provided by ADS.

\section{CONCLUSIONS}

In this article, we discuss a tuning-aided ISM optimization procedure. It utilizes the ADS tuning tool to estimate the sensitivities of various parameters. On

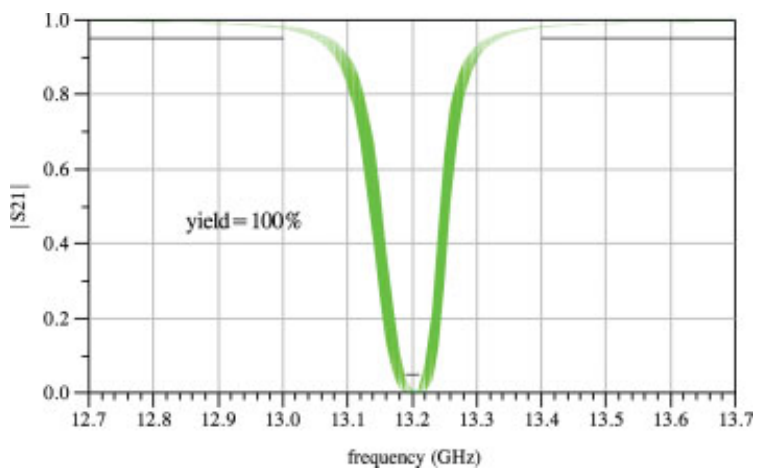

Figure 11. Yield estimation of the final surrogate using 1250 random points after Agilent ADS yield optimization. [Color figure can be viewed in the online issue, which is available at www.interscience.wiley.com.] 
the basis of this tuning process, we provide a guideline for choosing design variables or preassigned parameters, as well as for finding a good starting point for direct surrogate optimization and for parameter extraction of the surrogate. The proposed guideline increases the chances of an ISM algorithm to converge to a good fine model solution. We show that our method works well with a microstrip notch filter design.

\section{ACKNOWLEDGMENTS}

We thank Sonnet Software, Inc., Syracuse, NY, for $\boldsymbol{e m}^{\mathrm{TM}}$, and Agilent Technologies, Santa Rosa, CA, for ADS. J.W. Bandler is also with Bandler Corporation, Dundas, ON, Canada. This work was supported in part by the Natural Sciences and Engineering Research Council of Canada under Grants RGPIN7239-06 and STPGP336760-06, and by Bandler Corporation.

\section{REFERENCES}

1. J.W. Bandler, Q.S. Cheng, S.A. Dakroury, A.S. Mohamed, M.H. Bakr, K. Madsen, and J. Søndergaard, Space mapping: The state of the art, IEEE Trans Microwave Theory Tech 52 (2004), 337-361.

2. Q.S. Cheng, S. Koziel, and J.W. Bandler, Simplified space-mapping approach to enhancement of microwave device models, Int J RF Microwave ComputAided Eng 16 (2006), 518-535.
3. J.W. Bandler, Q.S. Cheng, N.K. Nikolova, and M.A. Ismail, Implicit space mapping optimization exploiting preassigned parameters, IEEE Trans Microwave Theory Tech 52 (2004), 378-385.

4. Q.S. Cheng and J.W. Bandler, An implicit space mapping technique for component modeling, Proc 36th European Microwave Conf, Manchester, UK, 2006, pp. 458-461.

5. Agilent Advanced Design System, Ver. 2005A, Agilent Technologies, Santa Rosa, CA, 2005.

6. Sonnet em, Ver. 10.53, Sonnet Software, North Syracuse, NY, 2005.

7. J.E. Rayas-Sánchez and V. Gutiérrez-Ayala, EMbased Monte Carlo analysis and yield prediction of microwave circuits using linear-input neural-output space mapping, IEEE Trans Microwave Theory Tech 54 (2006), 4528-4537.

8. M.S. Narayana and N. Gogia, Accurate design of a notch filter using electromagnetic simulator, Applied Microwave Wireless J 12 (2000), 44-48.

9. S. Koziel, J.W. Bandler, and K. Madsen, A spacemapping framework for engineering optimizationTheory and implementation, IEEE Trans Microwave Theory Tech 54 (2006), 3721-3730.

10. J.C. Rautio, RF design closure-Companion modeling and tuning methods, IEEE MTT-S Int Microwave Symp Workshop on Microwave Component Design Using Space Mapping Technology, San Francisco, CA, 2006.

11. D.G. Swanson Jr. and R.J. Wenzel, Fast analysis and optimization of combline filters using FEM, IEEE MTT-S Int. Microwave Symp Digest, Phoenix, AZ, 2001, pp. 1159-1162.

\section{BIOGRAPHIES}

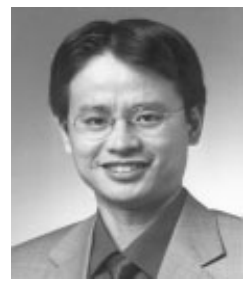

Qingsha (Shasha) Cheng was born in Chongqing, China. He received his B.Eng. degree and M.Eng. in automation from Chongqing University, Chongqing, China, in 1995 and 1998, respectively. $\mathrm{He}$ received his Ph.D. degree from McMaster University, Hamilton, Ontario, Canada, in 2004. Currently, he is a Research Associate in the Department of Electrical and Computer Engineering, McMaster University. He also serves as a Lecturer in the Faculty of Engineering at McMaster University.

In September 1998, he joined the Department of Computer Science and Technology, Peking University, Beijing, China. In September 1999, he joined the Simulation Optimization Systems Research Laboratory, Department of Electrical and Computer Engineering, McMaster University. His research interests are surrogate modeling, computer-aided design, design optimization and modeling of microwave devices, software design technology, and methodologies for microwave CAD. He has consulted for Bandler Corporation.

Dr. Qingsha Cheng has published nearly 20 papers in conference proceedings and journals. He was the recipient of the Nortel
Networks Ontario Graduate Scholarship in Science and Technology (OGSST) in 2001.

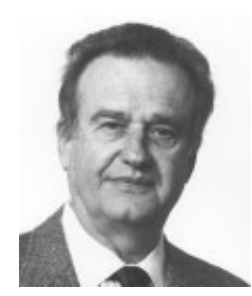

John W. Bandler was born in Jerusalem, on November 9, 1941. He studied at Imperial College of Science and Technology, London, England, from 1960 to 1966. He received the B.Sc.(Eng.), Ph.D., and D.Sc.(Eng.) degrees from the University of London, London, England, in 1963, 1967, and 1976, respectively. He joined Mullard Research Laboratories, Redhill, Surrey, England, in 1966. From 1967 to 1969 he was a Postdoctorate Fellow and Sessional Lecturer at the University of Manitoba, Winnipeg, Canada. Dr. Bandler joined McMaster University, Hamilton, Canada, in 1969. He has served as Chairman of the Department of Electrical Engineering and Dean of the Faculty of Engineering. He is currently Professor Emeritus in Electrical and Computer Engineering, directing research in the Simulation Optimization Systems Research Laboratory. 
Dr. Bandler was President of Optimization Systems Associates Inc. (OSA), which he founded in 1983, until November 20, 1997, the date of acquisition of OSA by Hewlett-Packard Company (HP). OSA implemented a first-generation yield-driven microwave CAD capability for Raytheon in 1985, followed by further innovations in linear and nonlinear microwave CAD technology for the Raytheon/Texas Instruments Joint Venture MIMIC Program. OSA introduced the CAE systems RoMPE ${ }^{\mathrm{TM}}$ in 1988 , $\mathrm{HarPE}^{\mathrm{TM}}$ in 1989, OSA90 ${ }^{\mathrm{TM}}$ and OSA90/hope ${ }^{\mathrm{TM}}$ in 1991, Empipe ${ }^{\mathrm{TM}}$ in 1992, Empipe3D ${ }^{\mathrm{TM}}$ and EmpipeExpress ${ }^{\mathrm{TM}}$ in 1996. OSA created the product $\boldsymbol{e m p a t h}^{\mathrm{TM}}$ in 1996 which was marketed by Sonnet Software, USA. Dr. Bandler is President of Bandler Corporation, which he founded in 1997.

Dr. Bandler was an Associate Editor of the IEEE Transactions on Microwave Theory and Techniques (1969-1974), and has continued serving as a member of the Editorial Board. He was Guest Editor of the Special Issue of the IEEE Transactions on Microwave Theory and Techniques on Computer-Oriented Microwave Practices (1974) and Guest Coeditor of the Special Issue of the IEEE Transactions on Microwave Theory and Techniques on Process-Oriented Microwave CAD and Modeling (1992). He joined the Editorial Boards of the International Journal of Numerical Modeling in 1987, the International Journal of Microwave and Millimeterwave Computer-Aided Engineering in 1989, and Optimization and Engineering in 1998. He was Guest Editor, International Journal of Microwave and Millimeter-Wave Computer-Aided Engineering, Special Issue on Optimization-Oriented Microwave CAD (1997), and Guest Editor, IEEE Transactions on Microwave Theory and Techniques, Special Issue on Automated Circuit Design Using Electromagnetic Simulators (1997). He was Guest Coeditor, Optimization and Engineering Special Issue on Surrogate Modeling and Space Mapping for Engineering Optimization (2001). He was Guest Coeditor, IEEE Transactions on Microwave Theory and Techniques, Special Issue on Electromagnetics-based Optimization of Microwave Components and Circuits (2004). He has served as Chair of the MTT-1 Technical Committee on Computer-Aided Design. Dr. Bandler has published more than 400 papers from 1965 to 2007 . He contributed to Modern Filter Theory and Design, Wiley-Interscience, 1973 and to Analog Methods for Computer-aided Analysis and Diagnosis, Marcel Dekker, 1988. Four of his papers have been reprinted in Computer-Aided Filter Design, IEEE Press, 1973, one in each of Microwave Integrated Circuits, Artech House, 1975; Low-Noise Microwave Transistors and Amplifiers, IEEE Press, 1981; Microwave Integrated Circuits, 2nd ed., Artech House, 1985; Statistical Design of Integrated Circuits, IEEE Press, 1987; and Analog Fault Diagnosis, IEEE Press, 1987.

Dr. Bandler is a Fellow of the Canadian Academy of Engineering, a Fellow of the Royal Society of Canada, a Life Fellow of the Institute of Electrical and Electronics Engineers, a Fellow of the Institution of Electrical Engineers (Great Britain), a Fellow of the Engineering Institute of Canada, a Member of the Association of Professional Engineers of the Province of Ontario (Canada), and a Member of the MIT Electromagnetics Academy. He received the Automatic Radio Frequency Techniques Group (ARFTG) Automated Measurements Career Award in 1994 and the IEEE MTT-S Microwave Application Award in 2004.

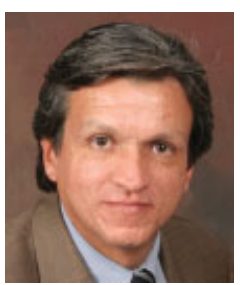

José Ernesto Rayas-Sánchez was born in Guadalajara, Jalisco, Mexico, on December 27, 1961. He received the B.Sc. degree in electronics engineering from the Instituto Tecnológico y de Estudios Superiores de Occidente (ITESO), Guadalajara, Mexico, in 1984, the Masters degree in electrical engineering from the Instituto Tecnológico y de Estudios Superiores de Monterrey (ITESM),

Monterrey, Mexico, in 1989, and the Ph.D. degree in electrical engineering from McMaster University, Hamilton, Ontario, Canada, in 2001.

Since 1989, he has been Full Professor in the Department of Electronics, Systems and Informatics, ITESO. In 1997, he began a sabbatical leave with the Simulation Optimization Systems Research Laboratory, McMaster University. In 2001, he returned to ITESO. He currently leads the Research Group on ComputerAided Engineering of Circuits and Systems (CAECAS) at ITESO. Since May 2005, he has been Profesor Numerario (honorary distinction) with ITESO. His research focuses on the development of novel methods and techniques for computer-aided and knowledge-based modeling, design, and optimization of high-speed electronic circuits and devices (including RF, microwave, and wireless circuits), exploiting highly accurate but computationally expensive simulators.

Dr. Rayas-Sánchez serves on the Editorial Boards of the IEEE Transactions on Microwave Theory and Techniques, the International Journal of RF and Microwave Computer-Aided Engineering (Wiley InterScience), and of the IEEE Latin America Transactions. He is also a member of the Technical Program Committee of the IEEE Microwave Theory and Techniques Society International Microwave Symposium (MTT-IMS), and a member of the IEEE Microwave Theory and Techniques Technical Committee on Computer Aided Design (MTT-1). During 2004 and 2005 he was the IEEE Mexican Council Chair, as well as the IEEE Region 9 Treasurer.

Dr. Rayas-Sánchez was the recipient of a 1997-2000 Consejo Nacional de Ciencia y Tecnología (CONACYT) scholarship presented by the Mexican Government, as well as a 2000-2001 Ontario Graduate Scholarship (OGS) presented by the Ministry of Training for Colleges and Universities in Ontario. He was also the recipient of a 2001-2003 CONACYT Repatriation and Installation Grants presented by the Mexican Government. He is the recipient of a 2004-2007 SEP-CONACYT Fundamental Scientific Research Grant presented by the Mexican Government. He is a member of the Mexican National System of Researchers, Level I. 